\title{
Nonlinear Decentralized Saturated Controller Design for Power Systems
}

\author{
Zairong Xi, Gang Feng, Senior Member, IEEE, Daizhan Cheng, Senior Member, IEEE, and Qiang Lu, Fellow, IEEE
}

\begin{abstract}
In this paper, a multimachine power system is first represented as the generalized Hamiltonian control system with dissipation. Then, a decentralized saturated steam valving and excitation controller, which is staticly measurable, is proposed based on the Hamiltonian function method. Last, an example of threemachine power system is discussed in detail.
\end{abstract}

Index Terms-Decentralized control, excitation control, Hamiltonian control system with dissipation, multimachine power system, saturated control, steam valving control.

\section{INTRODUCTION}

$\mathbf{R}$ ECENTLY, various advanced nonlinear control technologies have been applied to excitation and steam valving controllers of power systems [2], [7]-[10], [20]. Through careful investigation it is easy to see that most of these are based on differential geometric tools [5], which cancel the inherent system nonlinearities in order to obtain a feedback equivalent linear system. It has been shown in the literature that the dynamics of power systems can be exactly linearized by employing nonlinear feedback and a state transformation. One can then use the conventional linear control theory to design a controller in order to provide good performance [2], [6]-[11], [18]-[20]. However, these controllers suffer some flaws. First, it is well known that the amplitude of the controller is always bounded in the real world. In order to cope with the reality, the simulations in all of these researchers have used the bounded controller, which is the saturation of the original state feedback controller. But the validity of the saturation of the controller cannot be proved theoretically in these papers. Second, since physical limitation on the system structure makes information transfer among subsystems infeasible, decentralized controllers for multimachine power systems must be used in practice. However, the decentralized controller can not be obtained easily by these technologies because the controller must cancel the inherent system nonlinearities and a state transformation

Manuscript received January 24, 2001; revised November 3, 2002. Manuscript received in final form February 25, 2003. Recommended by Associate Editor A. Ray. This work is supported in part by the National Natural Science Foundation of China under Grant 59837270, the City University of Hong Kong under Grant SRG 7001245, the National Key Project of China under Grants G1998020308 and G1998020300, and the Institute of Systems Science, Academy of Mathematics and Systems Science, Chinese Academy of Sciences.

Z. Xi and D. Cheng are with Laboratory of Systems and Control, Institute of Systems Science, Academy of Mathematics and Systems Science, Chinese Academy of Sciences, Beijing, 100080, China (e-mail: zrxi@mail.iss.ac.cn).

G. Feng is with the Department of Manufacturing Engineering and Engineering Management, The City University of Hong Kong, Kowloon Tong, Hong Kong.

Q. Lu is with the Department of Electrical Engineering, Tsinghua University, Beijing, 100084, China.

Digital Object Identifier 10.1109/TCST.2003.813404 must be used in order to obtain a feedback equivalent linear system where the centralized information of the system has to be used. Thus, the following problem is very important in practice: how to design decentralized saturated controllers for multimachine power systems to improve the transient stability? It is an open problem as far as we know. It is also the most important motivation of this work. In order to solve the problem the structure information of the multimachine power systems should be used adequately and sufficiently.

Very recently, port-controlled Hamiltonian systems with dissipation (PCHD) have been extensively studied [3], [4], [12]-[15]. Indeed, the Hamiltonian function in PCHD systems is considered as the total energy (potential and kinetic energy) in the mechanical system and can play the role of a Lyapunov function for the system. In fact, a power system is an energy producing system, so it is convenient and natural to model the power system as a PCHD system. A single-machine infinite bus power system has been represented by a PCHD system [3], [16] and excitation control of a multimachine power system has also been written as a PCHD systems [21]. It has been shown from these researches that the Hamiltonian function method has some advantages.

In this paper, we shall concentrate on enhancing the transient stability of power systems by means of decentralized saturated nonlinear control. The resulting decentralized saturated nonlinear controller can guarantee the overall stability of a largescale power system. In fact, the most important and difficult step used in the controller design is to represent the considered system as Hamiltonian control system with dissipation, and then a saturated controller is easily obtained. The paper is arranged as follows. The fundamental result about the existence of a saturated controller for Hamiltonian system with dissipation is presented in view of convenience in Section II. In Section III, the applications of the proposed saturated controller to power systems are considered, and a slightly more realistic three-machine example system is also presented to illustrate the effectiveness of the proposed design method. Section IV gives some conclusions.

\section{PRELIMINARIES}

Consider the following port-controlled Hamiltonian systems with dissipation:

$$
\left\{\begin{array}{l}
\dot{x}=(J(x)-R(x)) \nabla H(x)+g(x) u, \quad x \in R^{n}, u \in R^{m} \\
y=g^{T}(x) \nabla H(x)
\end{array}\right.
$$

where $J(x)$ is a skew-symmetric structure matrix, $R(x)$ is a positive semidefinite matrix, the smooth function $H(x): R^{n} \rightarrow$ 
$R$ represents the total stored energy, which is called Hamiltonian function of the system (1), and $u, y \in R^{m}$ are the port power variables. The notation $\nabla H(x)$ is defined by $\nabla H(x)=$ $\left(\partial H / \partial x_{1}, \ldots, \partial H / \partial x_{n}\right)^{T}$.

The following theorem is our main result which is the basis of the paper.

Theorem 1: If the equilibrium point $x^{*}=0$ of Hamiltonian control system (1) is a strict local minimum, and system (1) is zero state detectable, then system (1) is stabilizable by a saturated output feedback control

$$
u_{i}=-L_{i} \operatorname{sat}\left(\frac{K_{i} y_{i}}{L_{i}}\right)
$$

where $L_{i}>0$ is the magnitude of saturation of the $i$ th control, $K_{i}>0$ is feedback gain parameter of the $i$ th control, and the saturated function $\operatorname{sat}(x)$ is defined as follows:

$$
\operatorname{sat}(x)= \begin{cases}1, & \text { if } x \geq 1 \\ x, & \text { if }-1<x<1 \\ -1, & \text { if } x \leq-1\end{cases}
$$

Proof: Taking $H(x)$ as a Lyapunov function, so

$$
\begin{aligned}
\frac{d H}{d t} & =-\nabla H^{T} R \nabla H+\nabla H^{T} g u \\
& =-\nabla H^{T} R \nabla H+\sum_{i=1}^{m} y_{i} u_{i} .
\end{aligned}
$$

It is easy to see that

$$
y_{i} u_{i}= \begin{cases}L_{i} y_{i}<0, & \text { if }-K_{i} y_{i} \geq L_{i} \\ -K_{i} y_{i} \leq 0, & \text { if }-L_{i}<-K_{i} y_{i}<L_{i} \\ -L_{i} y_{i}<0, & \text { if }-K_{i} y_{i} \leq-L_{i}\end{cases}
$$

and that

$$
y_{i} u_{i}=0
$$

is equivalent to

$$
y_{i}=0 .
$$

So we have

$$
\frac{d H}{d t} \leq 0
$$

and

$$
\frac{d H}{d t}=0
$$

implies

$$
y_{i}=0, \quad \forall i=1, \ldots, m .
$$

Then the trajectories of the closed-loop system converge to the largest invariant set contained in

$$
A=\{x: y \equiv 0, u \equiv 0\} .
$$

From the zero state detectability, the closed-loop system is asymptotically stable under the above saturated controller.

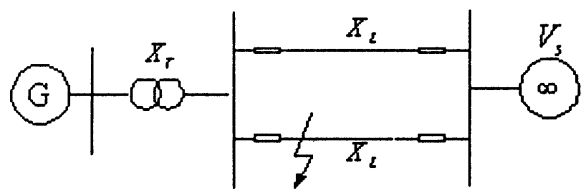

Fig. 1. Single machine infinite bus system.

Remark 1: As a matter of fact, the controller can be chosen as $u_{i}=-L_{i} \operatorname{sgn}\left(y_{i}\right)$ from the proof of above theorem which is a bang-bang control.

\section{APPLication to POWER Systems}

\section{A. Single Machine Infinite Bus Power Systems}

Consider a single machine infinite bus power system in this section. The system shown in Fig. 1 is modeled by the following dynamical system [1], [9]:

$$
\left\{\begin{aligned}
\dot{\delta}(t)= & \omega(t)-\omega_{0} \\
\dot{\omega}(t)= & -\frac{D}{H}\left(\omega(t)-\omega_{0}\right) \\
& +\frac{\omega_{0}}{H}\left(P_{H}+C_{M} P_{m 0}-\frac{V_{s}}{x_{d s}^{\prime}} E_{q}^{\prime} \sin (\delta(t))\right) \\
\dot{P}_{H}= & -\frac{1}{T_{H S}} P_{H}+\frac{C_{H}}{T_{H S}} P_{m 0}+\frac{C_{H}}{T_{H S}} u_{H} \\
\dot{E}_{q}^{\prime}= & -\frac{1}{T_{d}^{\prime}} E_{q}^{\prime}+\frac{1}{T_{d 0}} \frac{x_{d}-x_{d}^{\prime}}{x_{d s}^{\prime}} V_{s} \cos \delta+\frac{1}{T_{d 0}} V_{f}
\end{aligned}\right.
$$

where $\delta$ is the power angle between the $q$-axis electrical potential vector $\vec{E}_{q}$ and a reference bus voltage vector $\vec{V}_{\mathrm{REF}}$ in the system, in radians; $\omega$ is the rotating speed of the generator, in radians/second; $P_{H}$ is the mechanical power of high-pressure (HP) turbine, in per unit; $E_{q}^{\prime}$ are the $q$-axis internal transient electric potential of the generator, in per unit; $P_{m 0}$ is the initial mechanical power of the generator, in per unit; $H, C_{H}$ are moment of inertia in seconds and the power fraction of HP turbine, respectively; $T_{H S}=T_{H g}+T_{H}$ is the equivalent time constant of the HP turbine, $T_{H g}$ the time constant of oil-servomotor of regulated valve of the HP turbine, $T_{H}$ the time constant of HP turbine, $u_{H}$ the electrical control signal from the controller for the regulated valve, $T_{d}^{\prime}$ is the time constant of the field winding when the stator circuit is closed, $T_{d 0}$ is the excitation circuit time constant, $x_{d}$ is the $d$-axis synchronous reactance of a generator, $x_{d}^{\prime}$ is the $d$-axis transient reactance, and $V_{f}$ is the voltage of the field circuit of a generator.

Let $x_{1}=\delta(t), x_{2}=\omega(t)-\omega_{0}, x_{3}=P_{H}(t), x_{4}=E_{q}^{\prime}(t)$, and $a=D / H, b=\left(\omega_{0} / H\right) C_{M} P_{m 0}, c=\left(\omega_{0} / H\right)\left(V_{s} / x_{d s}^{\prime}\right)$, $d=1 / T_{H S}, e=\left(C_{H} / T_{H S}\right) P_{m 0}, h=\omega_{0} / H, p=1 / T_{d}^{\prime}$, $q=\left(1 / T_{d 0}\right)\left(x_{d}-x_{d}^{\prime} / x_{d s}^{\prime}\right) V_{s}$. Then (3) can be rewritten as follows:

$$
\left\{\begin{array}{l}
\dot{x}_{1}=x_{2} \\
\dot{x}_{2}=-a x_{2}+b+h x_{3}-c x_{4} \sin x_{1} \\
\dot{x}_{3}=-d x_{3}+e+\frac{C_{H}}{T_{H S}} u_{H} \\
\dot{x}_{4}=-p x_{4}+q \cos x_{1}+\frac{1}{T_{d 0}} V_{f} .
\end{array}\right.
$$


That is

$$
\begin{aligned}
& \frac{d}{d t}\left[\begin{array}{l}
x_{1} \\
x_{2} \\
x_{3} \\
x_{4}
\end{array}\right]=\left[\begin{array}{cccc}
0 & \frac{c p}{q} & 0 & 0 \\
-\frac{c p}{q} & -a \frac{c p}{q} & h K & 0 \\
0 & 0 & -d K & 0 \\
0 & 0 & 0 & -p
\end{array}\right] \\
& \times\left[\begin{array}{c}
\frac{q}{p} x_{4} \sin x_{1}-\frac{q}{c p} b-\frac{q}{p} \frac{h e}{c d} \\
\frac{q}{c p} x_{2} \\
\frac{1}{K}\left(x_{3}-\frac{e}{d}\right) \\
x_{4}-\frac{q}{p} \cos x_{1}
\end{array}\right]+\left[\begin{array}{cc}
0 & 0 \\
0 & 0 \\
\frac{C_{H}}{T_{H S}} u_{H} & 0 \\
0 & \frac{1}{T_{d 0}} V_{f}
\end{array}\right]
\end{aligned}
$$

where $K>0$ is a constant to be determined later.

It is easy to show that the matrix $-\left(M+M^{T}\right)$ is semipositive when

$$
K<\frac{4 a c p d}{h^{2} q}=\frac{4 D T_{d 0}}{\omega_{0} T_{H S} T_{d}^{\prime}\left(x_{d}-x_{d}^{\prime}\right)}
$$

where

$$
M=\left[\begin{array}{cccc}
0 & \frac{c p}{q} & 0 & 0 \\
-\frac{c p}{q} & -a \frac{c p}{q} & h K & 0 \\
0 & 0 & -d K & 0 \\
0 & 0 & 0 & -p
\end{array}\right] \text {. }
$$

Let

$$
\begin{aligned}
H(x)=\frac{1}{2} \frac{q}{c p} x_{2}^{2}-\frac{q}{c p}\left(b+\frac{h e}{d}\right) x_{1} & -\frac{q}{p} x_{4} \cos x_{1} \\
& +\frac{1}{2 K}\left(x_{3}-\frac{e}{d}\right)^{2}+\frac{1}{2} x_{4}^{2}
\end{aligned}
$$

which is bounded from below since $x_{1} \in[-\pi, \pi]$, and

$$
y=g^{T} \nabla H=\left[\begin{array}{c}
\frac{1}{K}\left(x_{3}-\frac{e}{d}\right) \\
x_{4}-\frac{q}{p} \cos x_{1}
\end{array}\right] .
$$

Thus, the system consisting of (3) and (4) is a generalized Hamiltonian control system with dissipation

$$
\left\{\begin{array}{l}
\dot{x}=(J(x)-R(x)) \nabla H(x)+g(x) u, \quad x \in R^{4}, u \in R^{2} \\
y=g^{T}(x) \nabla H(x)
\end{array}\right.
$$

where

$$
\begin{gathered}
J=\frac{M-M^{T}}{2}, \quad R=-\frac{M+M^{T}}{2}, g=\left[\begin{array}{llll}
0 & 0 & 1 & 0 \\
0 & 0 & 0 & 1
\end{array}\right]^{T} \\
x^{T}=\left[\begin{array}{llll}
x_{1} & x_{2} & x_{3} & x_{4}
\end{array}\right], \quad u=\left(\begin{array}{l}
u_{1} \\
u_{2}
\end{array}\right)=\left(\begin{array}{c}
\frac{C_{H}}{T_{H S}} u_{H} \\
\frac{1}{T_{d 0}} V_{f}
\end{array}\right) .
\end{gathered}
$$

Thus, the saturated controller

$$
\left\{\begin{array}{l}
u_{1}=-L_{1} \operatorname{sat}\left[\frac{K_{1} y_{1}}{L_{1}}\right]=-L_{1} \operatorname{sat}\left[\frac{K_{1}}{L_{1} K}\left(x_{3}-\frac{e}{d}\right)\right] \\
u_{2}=-L_{2} \operatorname{sat}\left[\frac{K_{2} y_{2}}{L_{2}}\right]=-L_{2} \operatorname{sat}\left[\frac{K_{2}}{L_{2}}\left(x_{4}-\frac{q}{p} \cos x_{1}\right)\right]
\end{array}\right.
$$

proposed via Theorem 1 should stabilize the system, where $L_{i}>0$ is the magnitude of saturation of the $i$ th control, $K_{i}>0$ is feedback gain parameter of the $i$ th control, $i=1,2$.

In the following, we verify the asymptotical stability of the closed-loop system. In fact, taking $H(x)$ as a Lyapunov function, we have

$$
\begin{aligned}
\frac{d H}{d t}= & -\frac{a q}{c p} x_{2}^{2}+h \frac{q}{c p} x_{2}\left(x_{3}-\frac{e}{d}\right)-\frac{d}{K}\left(x_{3}-\frac{e}{d}\right)^{2} \\
& -p\left(x_{4}-\frac{q}{p} \cos x_{1}\right)^{2}+y^{T} u \\
\leq & -\frac{a q}{c p}\left[x_{2}-\frac{h}{2 a}\left(x_{3}-\frac{e}{d}\right)\right]^{2} \\
& -\frac{4 a c p d-K q h^{2}}{4 a c p K}\left(x_{3}-\frac{e}{d}\right)^{2}-p\left(x_{4}-\frac{q}{p} \cos x_{1}\right)^{2} .
\end{aligned}
$$

Then the system is convergent to the biggest invariant subset of the set $A=\left\{x: x_{2}=0, x_{3}=e / d, x_{4}=(q / p) \cos x_{1}\right\}$. From $x_{2} \equiv 0, x_{3} \equiv e / d$ and $x_{4} \equiv(q / p) \cos x_{1}$, we know that $x_{1}=(1 / 2) \arcsin ((2(b d+h e) p) / c d q)$, which is exactly the equilibrium point of the system. Therefore, the system with trajectories contained in $A$ is asymptotically stable. From the La Salle invariant principle [17], the system is asymptotically stable. Then the following proposition is true.

Proposition 2: The excitation and steam valving control of single machine infinite bus power system model (3) has the following saturated stabilizing controller:

$$
\left\{\begin{array}{l}
u_{1}=L_{1} \text { sat }\left[-\frac{K_{1} y_{1}}{L_{1}}\right]=L_{1} \operatorname{sat}\left[-\frac{K_{1}}{L_{1} K}\left(x_{3}-\frac{e}{d}\right)\right] \\
u_{2}=L_{2} \text { sat }\left[-\frac{K_{2} y_{2}}{L_{2}}\right]=L_{2} \operatorname{sat}\left[-\frac{K_{2}}{L_{2}}\left(x_{4}-\frac{q}{p} \cos x_{1}\right)\right]
\end{array}\right.
$$

where $K_{1}>0, K_{2}>0, L_{1}>0$, and $L_{2}>0$ are the control gain and, respectively, the magnitude of saturation of the controls.

Remark 2: In fact, the controller (7) have the following form:

$$
\left\{\begin{array}{l}
u_{1}=L_{1} \mathrm{sat}\left[-\frac{K_{1}}{L_{1} K}\left(P_{H}(t)-C_{H} P_{m 0}\right)\right] \\
u_{2}=L_{2} \mathrm{sat}\left[-\frac{K_{2}}{L_{2}}\left(E_{q}^{\prime}(t)-\frac{T_{d 0} x_{d s}^{\prime}}{T_{d}^{\prime}\left(x_{d}-x_{d}^{\prime}\right) V_{s}} \cos \delta(t)\right)\right]
\end{array}\right.
$$

which can be directly measured. 


\section{B. Multimachine Power Systems}

Consider an $n$-machine power system in this section. The system can be modeled by the following equation [1], [9]:

$$
\left\{\begin{aligned}
\dot{\delta}_{i} & =\omega_{i}-\omega_{0} \\
\dot{\omega}_{i} & =-\frac{D_{i}}{H_{i}}\left(\omega_{i}-\omega_{0}\right)+\frac{\omega_{0}}{H_{i}}\left(P_{H i}+C_{M i} P_{m 0 i}-P_{e i}\right) \\
\dot{P}_{H i} & =-\frac{1}{T_{H S i}} P_{H i}+\frac{C_{H i}}{T_{H S i}} P_{m 0 i}+\frac{C_{H i}}{T_{H S i}} u_{H i} \\
\dot{E}_{q i}^{\prime} & =\frac{1}{T_{d 0 i}^{\prime}}\left(E_{f i}-E_{q i}\right)
\end{aligned}\right.
$$

and

$$
\begin{aligned}
E_{q i} & =E_{q i}^{\prime}+\left(x_{d i}-x_{d i}^{\prime}\right) I_{d i} \\
E_{f i} & =k_{c i} u_{f i} \\
I_{d i} & =-\sum_{j=1}^{n} E_{q i}^{\prime} B_{i j} \cos \left(\delta_{i}-\delta_{j}\right) \\
I_{q i} & =\sum_{j=1}^{n} B_{i j} E_{q j}^{\prime} \sin \left(\delta_{i}-\delta_{j}\right) \\
P_{e i} & =E_{q i}^{\prime} \sum_{j=1}^{n} B_{i j} E_{q j}^{\prime} \sin \left(\delta_{i}-\delta_{j}\right) \\
Q_{e i} & =-E_{q i}^{\prime} \sum_{j=1}^{n} B_{i j} E_{q j}^{\prime} \cos \left(\delta_{i}-\delta_{j}\right) \\
E_{q i} & =x_{a d i} I_{f i} \\
E_{q i} & =V_{t i}+\frac{Q_{e i} x_{d i}}{V_{t i}}
\end{aligned}
$$

where

$\delta_{i}$

$\omega_{i}$

$P_{H i}$

$E_{q i}^{\prime}$

$P_{m 0 i}$

$H_{i}$ and $C_{H i}$

$B_{i j}$

$P_{e i}$

$T_{H S i}=T_{H g i}+T_{H i}$

$T_{H g i}$ power angle between the $q$-axis electrical potential vector $\vec{E}_{q i}$ and a reference bus voltage vector $\vec{V}_{R E F}$ in the system in rad;

rotating speed of the $i$ th generator, in $\mathrm{rad} / \mathrm{s}$;

mechanical power of high-pressure (HP) turbine, in per unit; $q$-axis internal transient electric potential of the $i$ th generator, in per unit; initial mechanical power of the $i$ th generator, in per unit;

moment of inertia in second and the power fraction of HP turbine, respectively;

$i$ th row and $j$ th column element of

\begin{tabular}{|c|c|}
\hline$T_{H i}$ & time constant of HP turbine; \\
\hline$u_{H i}$ & $\begin{array}{l}\text { electrical control signal from the con- } \\
\text { troller for the regulated valve; }\end{array}$ \\
\hline$E_{q i}$ & EMF in the quadrature axis in per unit; \\
\hline$E_{f i}$ & $\begin{array}{l}\text { equivalent EMF in the excitation coil } \\
\text { in per unit. }\end{array}$ \\
\hline$T_{d 0 i}^{\prime}$ & $\begin{array}{l}\text { direct axis transient short circuit time } \\
\text { constant in seconds; }\end{array}$ \\
\hline$x_{d i}$ & $\begin{array}{l}\text { direct axis reactance of the } i \text { th gener- } \\
\text { ator in per unit; }\end{array}$ \\
\hline$x_{d i}^{\prime}$ & $\begin{array}{l}\text { direct axis transient reactance of the } i \text { th } \\
\text { generator, in per unit; }\end{array}$ \\
\hline$Q_{e i}$ & reactive power, in per unit \\
\hline$I_{f i}$ & excitation current, in per unit; \\
\hline$I_{d i}$ & direct axis current, in per unit; \\
\hline$I_{q i}$ & quadrature axis current, in per unit; \\
\hline$k_{c i}$ & $\begin{array}{l}\text { gain of the excitation amplifier, in per } \\
\text { unit; }\end{array}$ \\
\hline$u_{\text {fi }}$ & $\begin{array}{l}\text { input of the SCR amplifier of the } i \text { th } \\
\text { generator, in per unit; }\end{array}$ \\
\hline$x_{a d i}$ & $\begin{array}{l}\text { mutual reactance between the excita- } \\
\text { tion coil and the stator coil of the } i \text { th } \\
\text { generator, in per unit; }\end{array}$ \\
\hline$x_{T i}$ & transformer reactance; \\
\hline$x_{i j}$ & $\begin{array}{l}\text { transmission line reactance between } \\
\text { the } i \text { th generator and the } j \text { th generator; }\end{array}$ \\
\hline$V_{t i}$ & terminal voltage of the $i$ th generator. \\
\hline
\end{tabular}
the nodal susceptance matrix, which is symmetric, at the internal nodes after eliminating all physical buses, in per unit;

electric power;

equivalent time constant of HP turbine; time constant of oil-servomotor of regulated valve of HP turbine;
Remark 3: In power systems, $P_{e i}, Q_{e i}$, and $V_{t i}$ are readily measured. So $E_{q i}$ can be calculated using these available variables.

Denote $a_{i}=D_{i} / H_{i}, b_{i}=\omega_{0} / H_{i}, c_{i}=\left(\omega_{0} / H_{i}\right) C_{M i} P_{m 0 i}$, $d_{i}=\left(x_{d i}-x_{d i}^{\prime}\right) / T_{d 0 i}^{\prime}, e_{i}=1 / T_{H S i}, k_{i}=\left(C_{H i} / T_{H S i}\right) P_{m 0 i}$, $h_{i}=1 / T_{d 0 i}^{\prime}$. Let $x_{i 1}=\delta_{i}(t), x_{i 2}=\omega_{i}(t)-\omega_{0}, x_{i 3}=P_{H i}(t)$, and $x_{i 4}=E_{q i}^{\prime}(t)$ as state variables and $u_{i 1}=\left(C_{H i} / T_{H S i}\right) u_{H i}$ and $u_{i 2}=\left(1 / T_{d 0 i}^{\prime}\right) E_{f i}$ as controls. Then (9) can be rewritten as follows:

$$
\left\{\begin{aligned}
\dot{x}_{i 1}= & x_{i 2} \\
\dot{x}_{i 2}= & -a_{i} x_{i 2}+c_{i}+b_{i} x_{i 3}-b_{i} x_{i 4} \sum_{j=1}^{n} B_{i j} x_{j 4} \\
& \times \sin \left(x_{i 1}-x_{j 1}\right) \\
\dot{x}_{i 3}= & -e_{i} x_{i 3}+k_{i}+u_{i 1} \\
\dot{x}_{i 4}= & -h_{i} x_{i 4}+d_{i} \sum_{j=1}^{n} B_{i j} x_{j 4} \cos \left(x_{i 1}-x_{j 1}\right)+u_{i 2} .
\end{aligned}\right.
$$

It is easy to see that the system (10) can be represented as

$$
\begin{aligned}
\frac{d}{d t}\left[\begin{array}{l}
x_{i 1} \\
x_{i 2} \\
x_{i 3} \\
x_{i 4}
\end{array}\right]=\left[\begin{array}{cccc}
0 & b_{i} & 0 & 0 \\
-b_{i} & -a_{i} b_{i} & b_{i} K_{i} & 0 \\
0 & 0 & -e_{i} K_{i} & 0 \\
0 & 0 & 0 & -d_{i}
\end{array}\right] \nabla_{x_{i}} H \\
+\left[\begin{array}{ll}
0 & 0 \\
0 & 0 \\
1 & 0 \\
0 & 1
\end{array}\right]\left[\begin{array}{l}
u_{i 1} \\
u_{i 2}
\end{array}\right]
\end{aligned}
$$


where $x_{i}^{T}=\left(x_{i 1}, x_{i 2}, x_{i 3}, x_{i 4}\right), K_{i}>0$ is a constant and

$$
\begin{aligned}
H=\sum_{i=1}^{n}\left[\frac{1}{2 b_{i}} x_{i 2}^{2}-\left(\frac{c_{i}}{b_{i}}+\frac{k_{i}}{e_{i}}\right) x_{i 1}+\frac{1}{2 K_{i}}\left(x_{i 3}-\frac{k_{i}}{e_{i}}\right)^{2}\right. \\
\left.-\frac{1}{2} x_{i 4} \sum_{j=1}^{n} B_{i j} x_{j 4} \cos \left(x_{i 1}-x_{j 1}\right)+\frac{h_{i}}{2 d_{i}} x_{i 4}^{2}\right] .
\end{aligned}
$$

Given an operation point $\left(x_{i 1}^{e}, 0, x_{i 3}^{e}, x_{i 4}^{e}\right)$, one must insert constant controls $\bar{u}_{i 1}$ and $\bar{u}_{i 2}$ such that

$$
\left\{\begin{array}{l}
x_{i 2}^{e}=0 \\
c_{i}+b_{i} x_{i 3}^{e}=b_{i} x_{i 4}^{e} \sum_{j=1}^{n} B_{i j} x_{j 4}^{e} \sin \left(x_{i 1}^{e}-x_{j 1}^{e}\right) \\
x_{i 3}^{e}=\frac{k_{i}+\bar{u}_{i 1}}{e_{i}} \\
x_{i 4}^{e}=\frac{d_{i}}{h_{i}} \sum_{j=1}^{n} B_{i j} x_{j 4}^{e} \cos \left(x_{i 1}^{e}-x_{j 1}^{e}\right)+\bar{u}_{i 2} .
\end{array}\right.
$$

Let

$$
\begin{aligned}
H_{e}=\sum_{i=1}^{n} & {\left[\frac{1}{2 b_{i}} x_{i 2}^{2}-\left(\frac{c_{i}}{b_{i}}+\frac{k_{i}+\bar{u}_{i 1}}{e_{i}}\right) x_{i 1}\right.} \\
& +\frac{1}{2 K_{i}}\left(x_{i 3}-\frac{k_{i}+\bar{u}_{i 1}}{e_{i}}\right)^{2}+\frac{h_{i}}{2 d_{i}} x_{i 4}^{2}-\frac{\bar{u}_{i 2}}{d_{i}} x_{i 4} \\
& \left.-\frac{1}{2} x_{i 4} \sum_{j=1}^{n} B_{i j} x_{j 4} \cos \left(x_{i 1}-x_{j 1}\right)\right]
\end{aligned}
$$

which is bounded from below because of $x_{i 1} \in[-\pi, \pi]$, and

$$
\begin{aligned}
y_{i} & =\left(\begin{array}{llll}
0 & 0 & 1 & 0 \\
0 & 0 & 0 & 1
\end{array}\right) \nabla_{x_{i}} H_{e} \\
& =\left(\begin{array}{c}
\frac{1}{K_{i}}\left(x_{i 3}-\frac{k_{i}+\bar{u}_{i 1}}{e_{i}}\right) \\
\frac{h_{i}}{d_{i}} x_{i 4}-\sum_{j=1}^{n} B_{i j} x_{j 4} \cos \left(x_{i 1}-x_{j 1}\right)-\frac{\bar{u}_{i 2}}{d_{i}}
\end{array}\right) .
\end{aligned}
$$

Then the forced system (10)-(12) with the feedback control

$$
u_{i j}=\bar{u}_{i j}+v_{i j}, \quad i=1, \ldots, n, j=1,2
$$

can be represented as

$$
\begin{aligned}
\frac{d}{d t}\left[\begin{array}{l}
x_{i 1} \\
x_{i 2} \\
x_{i 3} \\
x_{i 4}
\end{array}\right]= & {\left[\begin{array}{cccc}
0 & b_{i} & 0 & 0 \\
-b_{i} & -a_{i} b_{i} & b_{i} K_{i} & 0 \\
0 & 0 & -e_{i} K_{i} & 0 \\
0 & 0 & 0 & -d_{i}
\end{array}\right] \nabla_{x_{i}} H_{e} } \\
& +\left[\begin{array}{ll}
0 & 0 \\
0 & 0 \\
1 & 0 \\
0 & 1
\end{array}\right]\left[\begin{array}{l}
v_{i 1} \\
v_{i 2}
\end{array}\right] \\
y_{i}= & \left(\begin{array}{llll}
0 & 0 & 1 & 0 \\
0 & 0 & 0 & 1
\end{array}\right) \nabla_{x_{i}} H_{e}
\end{aligned}
$$

It is easy to see that

$$
\begin{aligned}
R_{i} & =-\frac{1}{2}\left(\left[\begin{array}{cccc}
0 & b_{i} & 0 & 0 \\
-b_{i} & -a_{i} b_{i} & b_{i} K_{i} & 0 \\
0 & 0 & -e_{i} K_{i} & 0 \\
0 & 0 & 0 & -d_{i}
\end{array}\right]\right. \\
& \left.+\left[\begin{array}{cccc}
0 & b_{i} & 0 & 0 \\
-b_{i} & -a_{i} b_{i} & b_{i} K_{i} & 0 \\
0 & 0 & -e_{i} K_{i} & 0 \\
0 & 0 & 0 & -d_{i}
\end{array}\right]^{T}\right) \\
& =\left(\begin{array}{cccc}
0 & 0 & 0 & 0 \\
0 & a_{i} b_{i} & -\frac{b_{i} K_{i}}{2} & 0 \\
0 & -\frac{b_{i} K_{i}}{2} & e_{i} K_{i} & 0 \\
0 & 0 & 0 & d_{i}
\end{array}\right) \geq 0
\end{aligned}
$$

when $K_{i}<4 a_{i} e_{i} / b_{i}=4 D_{i} / \omega_{0} T_{H S i}$. That is to say, when $K_{i}<4 a_{i} e_{i} / b_{i}$ the system (13) is a generalized Hamiltonian control system with dissipation.

Then the following saturated controller is designed:

$$
\left\{\begin{aligned}
& v_{i 1}=-L_{i 1} \operatorname{sat}\left(\frac{l_{i 1} y_{i 1}}{L_{i 1}}\right) \\
&=-L_{i 1} \operatorname{sat}\left(\frac{l_{i 1}}{L_{i 1} K_{i}}\left(x_{i 3}-\frac{k_{i}+\bar{u}_{i 1}}{e_{i}}\right)\right) \\
& v_{i 2}=-L_{i 2} \operatorname{sat}\left(\frac{l_{i 2} y_{i 2}}{L_{i 2}}\right) \\
&=-L_{i 2} \operatorname{sat}\left(\frac{l_{i 2}}{L_{i 2}}\left(\frac{h_{i}}{d_{i}} x_{i 4}-\frac{\bar{u}_{i 2}}{d_{i}}\right)\right. \\
&\left.-\frac{l_{i 2}}{L_{i 2}} \sum_{j=1}^{n} B_{i j} x_{j 4} \cos \left(x_{i 1}-x_{j 1}\right)\right) \\
& i=1, \ldots, n
\end{aligned}\right.
$$

where $l_{i 1}>0$ and $l_{i 2}>0$ are control gains which can be arbitrarily selected and $L_{i 1}>0$ and $L_{i 2}>0$ are magnitude of saturation of the controls which can be arbitrarily selected also. Selecting $H_{e}(x)$ as a Lyapunov function, we have

$$
\begin{aligned}
\frac{d H}{d t} & =-\sum_{i=1}^{n}\left(\nabla_{x_{i}} H_{e}^{T} R_{i} \nabla_{x_{i}} H_{e}+y_{i 1} v_{i 1}+y_{i 2} v_{i 2}\right) \\
& \leq-\sum_{i=1}^{n} \nabla_{x_{i}} H_{e}^{T} R_{i} \nabla_{x_{i}} H_{e}
\end{aligned}
$$

Then, the closed-loop system is convergent to the largest invariant set contained in

$$
\begin{gathered}
A=\left\{x: x_{i 2}^{e}=0, x_{i 3}^{e}=\frac{k_{i}+\bar{u}_{i 1}}{e_{i}},\right. \\
\left.x_{i 4}^{e}=\frac{d_{i}}{h_{i}} \sum_{j=1}^{n} B_{i j} x_{j 4}^{e} \cos \left(x_{i 1}^{e}-x_{j 1}^{e}\right)+\bar{u}_{i 2}\right\} .
\end{gathered}
$$


From $x_{i 2} \equiv 0, x_{i 3} \equiv k_{i} / e_{i}$, and $x_{i 4}^{e}=\left(d_{i} / h_{i}\right)$ $\sum_{j=1}^{n} B_{i j} x_{j 4}^{e} \cos \left(x_{i 1}^{e}-x_{j 1}^{e}\right)+\bar{u}_{i 2}$, we know that $c_{i}+b_{i} x_{i 3}^{e}=b_{i} x_{i 4}^{e} \sum_{j=1}^{n} B_{i j} x_{j 4}^{e} \sin \left(x_{i 1}^{e}-x_{j 1}^{e}\right)$, which is exactly the equilibrium point of the closed-loop system. Therefore, the closed-loop system with trajectories contained in $A$ is asymptotically stable. From the La Salle invariant principle [17], the closed-loop system is asymptotically stable. So, the following proposition is true.

Proposition 3: The multimachine power system model (9) has the following saturated stabilizing controller:

$$
\left\{\begin{array}{c}
u_{H i}=\frac{T_{H S i}}{C_{H i}}\left[\bar{u}_{i 1}-L_{i 1} \operatorname{sat}\left(\frac{l_{i 1}}{L_{i 1} K_{i}}\left(x_{i 3}-\frac{k_{i}+\bar{u}_{i 1}}{e_{i}}\right)\right)\right] \\
E_{f i}=T_{d 0 i}^{\prime}\left[\bar{u}_{i 2}-L_{i 2} \operatorname{sat}\left(\frac{l_{i 2}}{L_{i 2}}\left(\frac{h_{i}}{d_{i}} x_{i 4}-\frac{\bar{u}_{i 2}}{d_{i}}\right)\right.\right. \\
\left.\left.-\frac{l_{i 2}}{L_{i 2}} \sum_{j=1}^{n} B_{i j} x_{j 4} \cos \left(x_{i 1}-x_{j 1}\right)\right)\right] \\
i=1, \ldots, n
\end{array}\right.
$$

around a prescribed operation point $\left(x_{i 1}^{e}, 0, x_{i 3}^{e}, x_{i 4}^{e}\right)$, where $l_{i j}>0(i=1, \ldots, n, j=1,2)$ are the control gains and $L_{i j}>$ $0(i=1, \ldots, n, j=1,2)$ are the magnitude of saturation of the control.

Remark 4: It is easy to see that

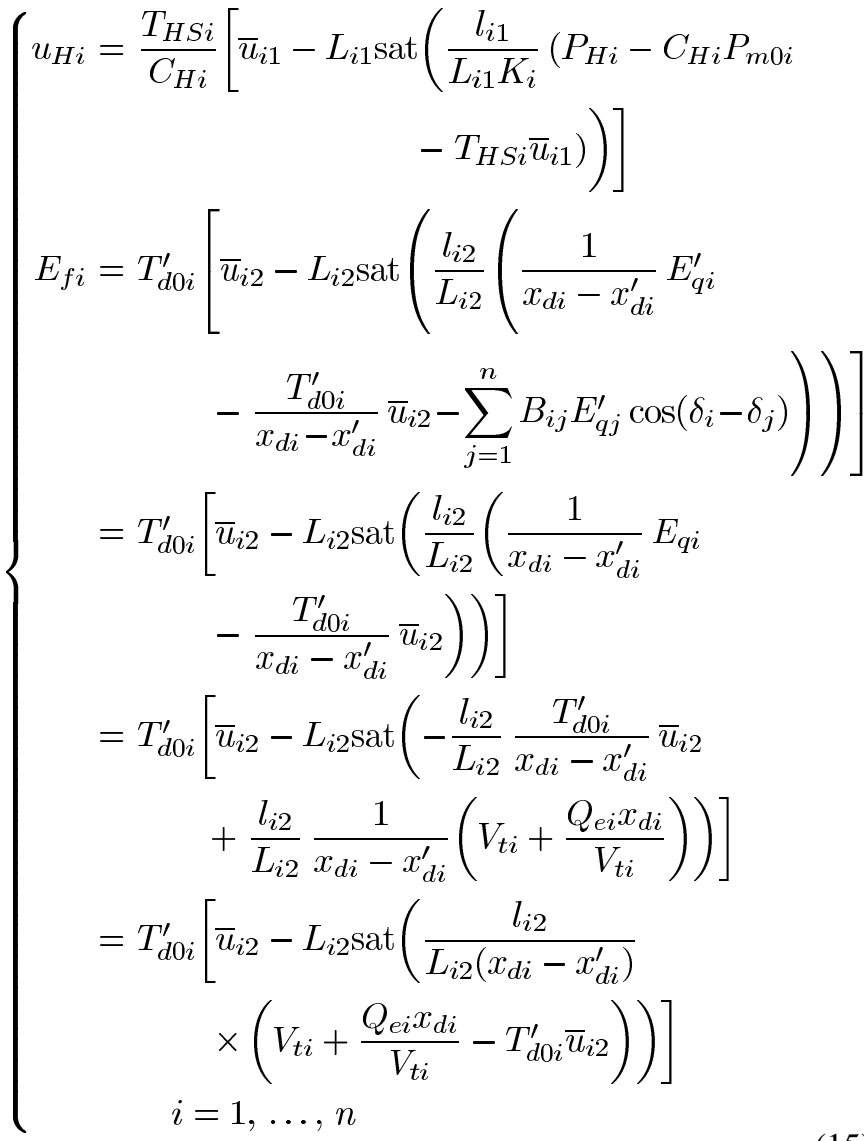

which is decentralized and can be directly measured. That is, the controller (15) is decentralized, saturated, and measurable.

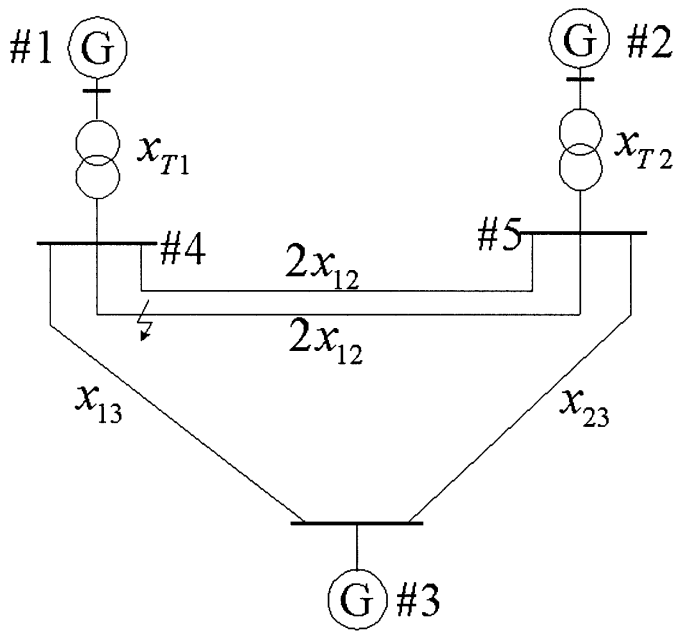

Fig. 2. Three-machine example system.

\section{Three-Machine Power System Example}

A three-machine example system shown in Fig. 2 is chosen to demonstrate the effectiveness of the proposed decentralized saturated controller.

The system parameters used in the simulation are as follows

\begin{tabular}{|c|c|c|}
\hline System parameters & Generator \#1 & Generator \#2 \\
\hline$x_{d}$ (per unit) & 1.863 & 2.36 \\
\hline$x_{d}^{\prime}$ (per unit) & 0.257 & 0.319 \\
\hline$x_{T}$ (per unit) & 0.129 & 0.11 \\
\hline$D$ (per unit) & 5 & 3 \\
\hline$T_{d 0}^{\prime}$ (per unit) & 6.9 & 7.96 \\
\hline$H(\mathrm{~s})$ & 8 & 10.2 \\
\hline$x_{a d}(\mathrm{~s})$ & 1.712 & 1.712 \\
\hline$k_{c}$ & 1 & 1 \\
\hline$\omega_{0}(\mathrm{rad} / \mathrm{s})$ & 314.159 & \\
\hline$C_{M}$ & 0.7 & 0.72 \\
\hline$C_{H}$ & 0.3 & 0.29 \\
\hline$P_{m 0}$ & 0.82 & 0.8 \\
\hline$T_{H \sum i}$ & 0.398 & 0.4 \\
\hline$x_{12}$ & \multicolumn{2}{|c|}{0.55} \\
\hline$x_{13}$ & \multicolumn{2}{|c|}{0.53} \\
\hline$x_{23}$ & \multicolumn{2}{|c|}{0.6} \\
\hline
\end{tabular}
[20]:

In the example system, the generator \#3 is chosen as slack bus. In simulation the control parameters are selected as $l_{11}=$ $0.02, l_{12}=0.01, l_{21}=0.03, l_{22}=0.02, K_{1}=0.05$, and $K_{2}=0.01$. Suppose the operation point is given as $(0.2700,0$, $0.2460,2.2257,0.2500,0,0.2320,3.4379)$, then one can obtain $\bar{u}_{11}=0, \bar{u}_{12}=0.5720, \bar{u}_{21}=0$, and $\bar{u}_{22}=1.2190$. The control input limitations are supposed to be

$$
\begin{aligned}
& -0.05 \leq u_{i 1}-\bar{u}_{i 1} \leq 0.05, \\
& -0.01 \leq u_{i 2}-\bar{u}_{i 2} \leq 0.01, \quad i=1,2 .
\end{aligned}
$$

The simulation results concerning the dynamic behavior of the three-machine system under the proposed control law are shown in Figs. 3-6, where a typical symmetrical three-phase short-circuit fault occurring on the transmission line near bus 4 is considered. 


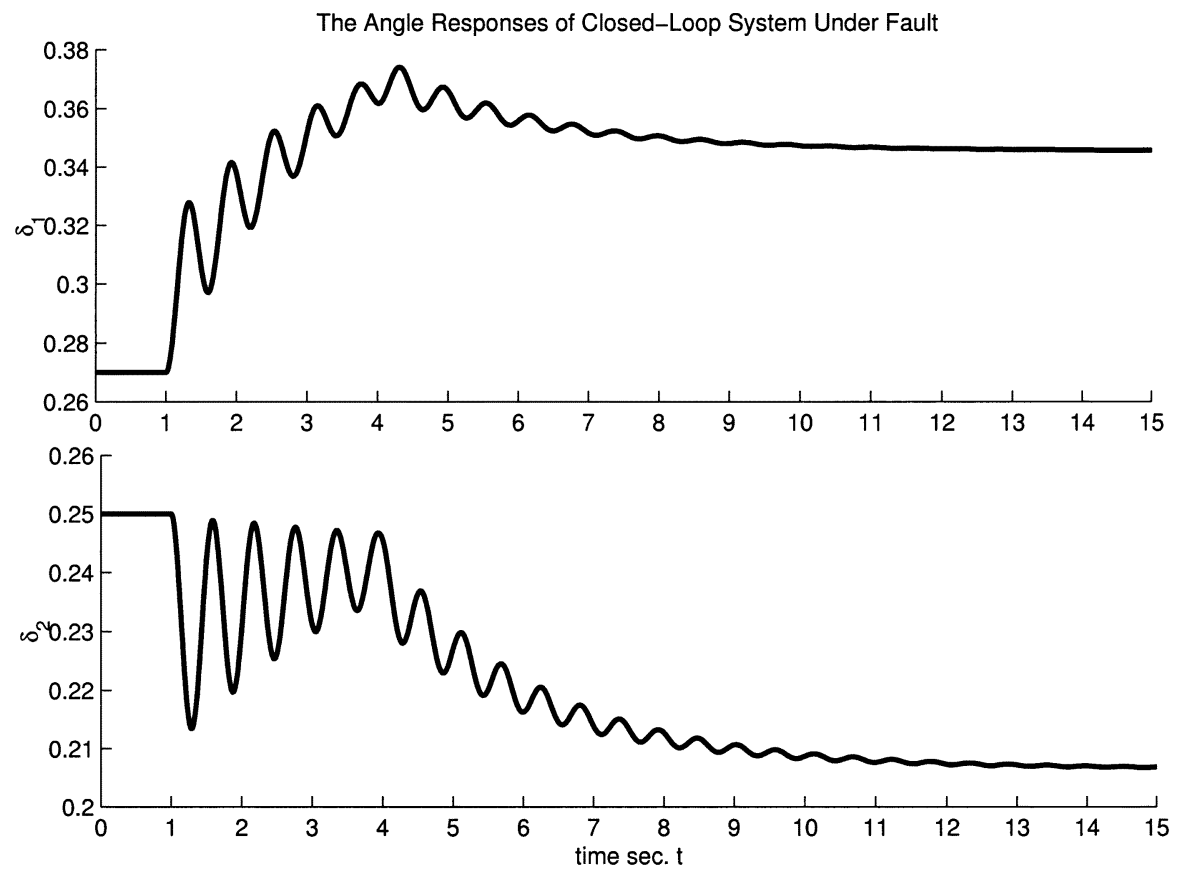

Fig. 3. Responses of angles $\delta_{1}$ and $\delta_{2}$ under the fault that occurs at $1 \mathrm{~s}$ and is cleared at $4 \mathrm{~s}$.
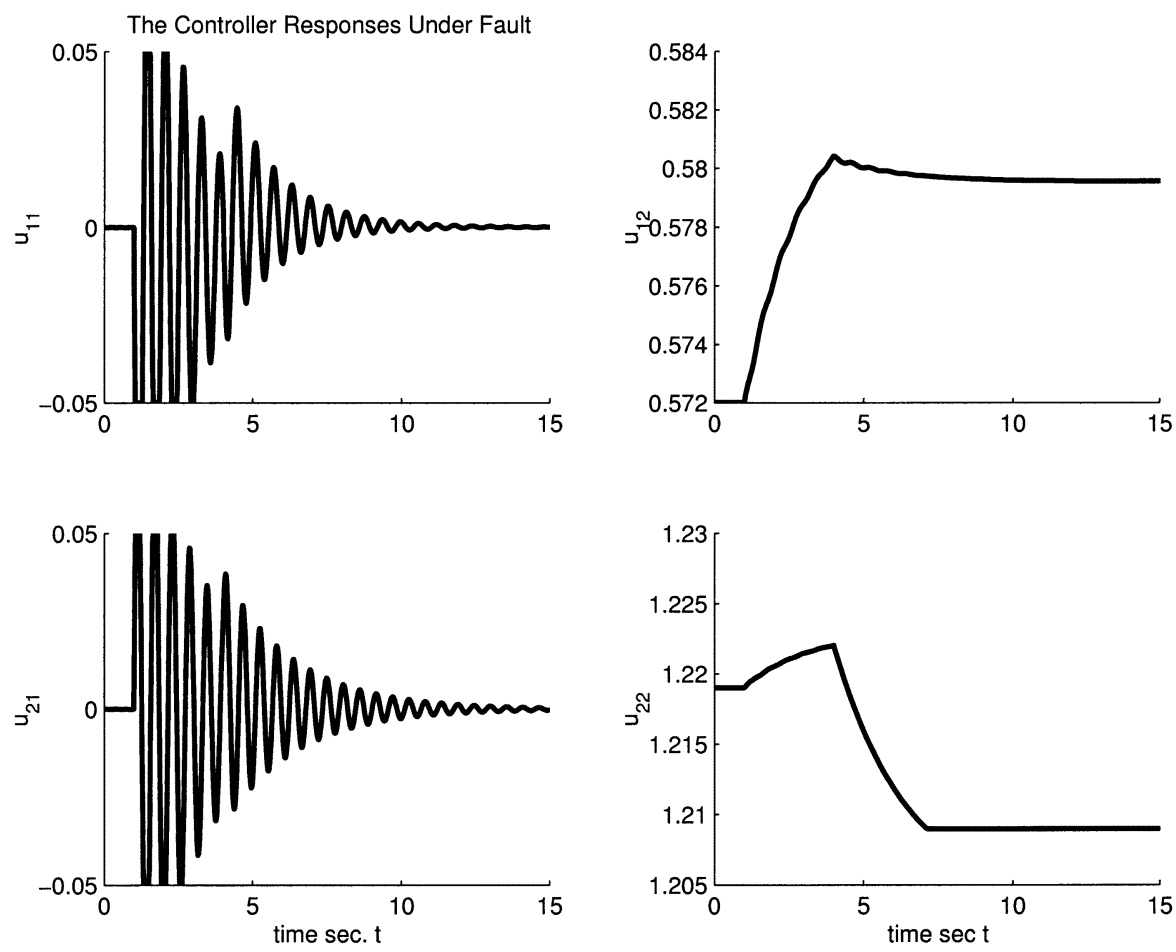

Fig. 4. Responses of the controller $u_{11}, u_{12}, u_{21}$, and $u_{22}$ under the fault that occurs at $1 \mathrm{~s}$ and is cleared at $4 \mathrm{~s}$.

It can be observed from Figs. 3 and 4 that with the proposed saturated decentralized control law the dynamic system is stabilized under the fault that occurs at $1 \mathrm{~s}$ and is cleared at $4 \mathrm{~s}$.

It can be further observed from Figs. 5 and 6 that with the proposed saturated decentralized control law the dynamic system is also stabilized under the fault that occurs at $1 \mathrm{~s}$, is cleared at 4 $\mathrm{s}$, and restored at $5 \mathrm{~s}$.

Comparing Figs. 3 and 4 with Figs. 5 and 6, one notices that the resulting operation point can be restored if the transmission line is restored. This indicates that restoring transmission line is useful for the dynamical performance of closed-loop system.

\section{CONCLUSION}

In this paper, the controller design of power systems have been considered. Using Hamiltonian function method a decentralized, saturated, and measurable controller has been obtained. In fact, in the literature decentralized saturated controllers are 

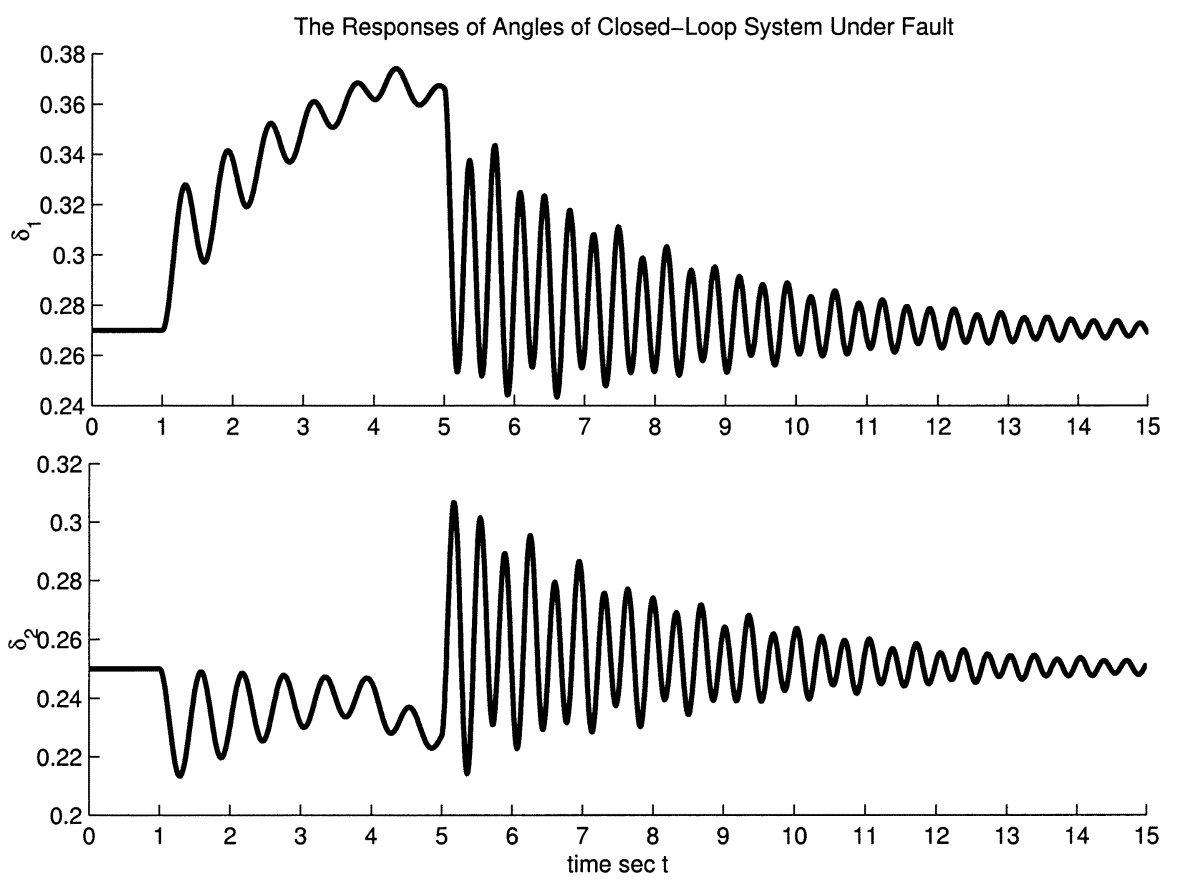

Fig. 5. The responses of angles $\delta_{1}$ and $\delta_{2}$ under the fault that occurs at $1 \mathrm{~s}$, cleared at $4 \mathrm{~s}$, and restored at $5 \mathrm{~s}$.
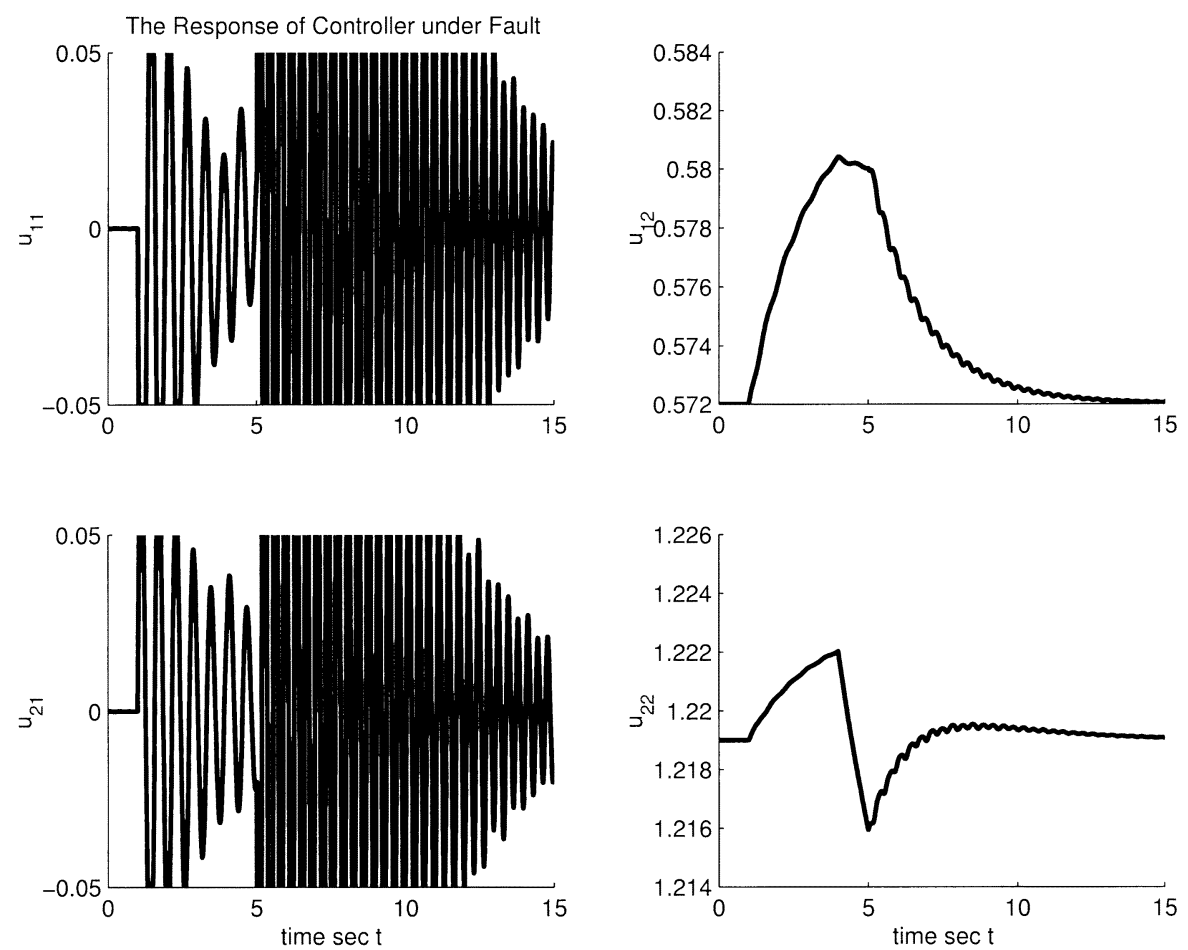

Fig. 6. Responses of the controller $u_{11}, u_{12}, u_{21}$, and $u_{22}$ under the fault that occurs at $1 \mathrm{~s}$, cleared at $4 \mathrm{~s}$, and restored at $5 \mathrm{~s}$.

widely used for power systems, but no theoretic foundation is given. The result of this paper have provided theoretic foundation. Finally, a three-machine power system has been presented to illustrate the proposed controller design.

\section{ACKNOWLEDGMENT}

The authors wish to acknowledge the help of Dr. M. Bodson, Editor-in-Chief, and W. Johnson. The authors wish to thank the anonymous referees for careful reading of the manuscript. Their helpful comments are greatly appreciated.

\section{REFERENCES}

[1] A. R. Bergen, Power Systems Analysis. Englewood Cliffs, NJ: Prentice-Hall, 1986

[2] J. W. Chapman, M. D. IliC, C. A. King, L. Eng, and H. Kaufman, "Stabilizing a multimachine power system via decentralized feedback linearizing excitation control," IEEE Trans. Power Syst., vol. 8, pp. 830-839, Aug. 1993. 
[3] D. Cheng, Z. Xi, Y. Hong, and H. Qin, "Energy-based stabilization of forced Hamiltonian systems with its application to power systems," in Proc. 14th IFAC, 1999, pp. 297-302.

[4] G. Escobar, A. J. van der Schaft, and R. Ortega, "A Hamiltonian viewpoint in the modeling of switching power converters," Automatica, vol. 35, pp. 445-452, 1999 .

[5] A. Isidori, "Nonlinear control systems: An introduction," in Communications and Control Engineering, 3rd ed. New York: Springer-Verlag, 1995.

[6] S. Jain, F. Khorrami, and B. Fardanesh, "Adaptive nonlinear excitation control of power system with unknown interconnections," IEEE Trans. Control Syst. Technol., vol. 2, pp. 436-446, Dec. 1994.

[7] S. Jain and F. Khorrami, "Robust decentralized control of power systems utilizing only swing angle measurements," Int. J. Contr., vol. 66, pp. 581-601, 1997.

[8] C. A. King, J. W. Chapman, and M. D. Ilic, "Feedback linearizing excitation control on a full-scale power system model," IEEE Trans. Power Syst., vol. 9, pp. 1102-1109, May 1994.

[9] Q. Lu and Y. Sun, "Nonlinear stabilization control of multimachine systems," IEEE Trans. Power Syst., vol. 4, pp. 236-241, Feb. 1989.

[10] Q. Lu, Y. Sun, Z. Xu, and T. Mochizuki, "Decentralized nonlinear optimal excitation control," IEEE Trans. Power Syst., vol. 11, pp. 1957-1962, Nov. 1996.

[11] F. K. Mark, "Design of nonlinear generator excitors using differential geometric control theories," in Proc. 31st IEEE Conf. Decision Contr., Tucson, AZ, 1992, pp. 1149-1153.

[12] B. M. J. Maschke, R. Ortega, and A. J. van der Schaft, "Energy-based Lyapunov functions for forced dissipation," in Proc. 37th IEEE Conf. Decision Contr., 1998, pp. 3599-3604.

[13] B. M. J. Maschke, R. Ortega, A. J. van der Schaft, and G. Escobar, "An energy-based derivation of Lyapunov functions for forced systems with application to stabilizing control," in Proc. 14th IFAC, Beijing, China, 1999.

[14] R. Ortega, A. Loría, P. J. Nicklasson, and H. Sira-Ramírez, "Passivitybased control of Euler-Lagranigan systems," in Communications and Control Engineering. London, U.K.: Springer-Verlag, 1998.

[15] R. Ortega, A. J. van der Schaft, B. M. J. Maschke, and G. Escobar, "Energy-shaping of port-controlled Hamiltonian systems by interconnection," in Proc. 38th IEEE Conf. Decision Contr., 1999, pp. 1646-1651.

[16] R. Ortega, M. Galaz-Larios, A. S. Bazanella, and A. Stankovic, "Excitation control of synchronous generators via total energy-shaping," in Proc. Amer. Contr. Conf., Arlington, VA, 2001, pp. 817-822.

[17] J. J. E. Slotine and W. Li, Applied Nonlinear Control. Englewood Cliffs, NJ: Prentice-Hall, 1991.

[18] C. Sun, Z. Zhao, Y. Sun, and Q. Lu, "Design of nonlinear robust excitation control for multimachine power systems," in Proc. Inst. Elect. Eng. Generation, Transmission, and Distribution, vol. 143, 1996, pp. 253-257.

[19] Y. Wang, L. Xie, D. J. Hill, and R. H. Middleton, "Robust nonlinear controller design for transient stability enhancement of power systems," in Proc. 31st IEEE Conf. Decision Contr., Tuscon, AZ, 1992, pp. $1117-1122$.

[20] Y. Wang, G. Guo, and D. J. Hill, "Robust decentralized nonlinear controller design for multimachine power systems," Automatica, vol. 33, no. 9, pp. 1725-1733, 1997.

[21] Z. Xi, D. Cheng, Q. Lu, and S. Mei, "Nonlinear decentralized controller design for multimachine power systems using Hamiltonian function method," Automatica, vol. 38, no. 3, pp. 527-534, 2002.

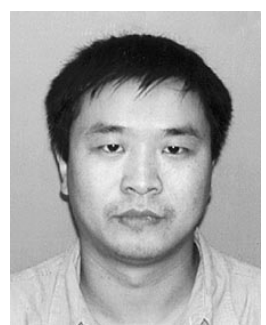

Zairong Xi was born in Hunan, China, in 1969. He received the M.S. degree in mathematics from Zhengzhou University, Zhengzhou, China, in 1997, and the Ph.D. degree in control theory from the Chinese Academy of Sciences, Beijing, China, in 2000.

He was a Postdoctoral Fellow in Tsinghua University, Beijing. In 2002, he joined the Laboratory of Systems and Control, Institute of Systems Science, Academy of Mathematics and Systems Science, Chinese Academy of Sciences. His research interests include nonlinear systems analysis and synthesis, power systems control, mechanical systems, stability analysis, and robust control.

Dr. Xi received the Excellent Prize of the President Scholarship and Prize of Huawei from the Chinese Academy of Sciences in 2000.

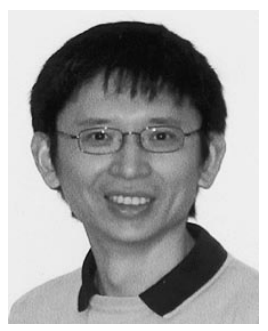

Gang Feng (S'90-M'92-SM'95) received the B.Eng. and M.Eng. degrees in automatic control of electrical engineering from the Nanjing Aeronautical Institute, Nanjing, China, in 1982 and 1984, respectively, and the Ph.D. degree in electrical engineering from the University of Melbourne, Melbourne, Australia, in 1992.

From 1992 to 1999, he was with School of Electrical Engineering, University of New South Wales, Adelaide, Australia. Since 2000, he has been with City University of Hong Kong, Hong Kong. In 1997, he was a Visiting Fellow with the National University of Singapore, Singapore, and a Visiting Fellow in 1997-1998 with the Aachen Technology University, Germany. His current research interests include robust adaptive control, robot control, and intelligent control. He has authored and/or coauthored more than 170 referred technical papers.

Dr. Feng is an Associate Editor of IEEE TRANSACTIONS ON FUZZY SYSTEMS and was an Associate Editor of the Conference Editorial Board of IEEE Control System Society. He was awarded an Alexander von Humboldt Fellowship in 1997-1998.

Daizhan Cheng (SM'02) received the Bachelor's degree from Tsinghua University, Beijing, China, in 1970, the M.S. degree from the Chinese Academy of Sciences, Beijing, in 1981, and the Ph.D. degree from Washington University, St. Louis, MO, in 1985.

Since 1990, he has been a Professor with the Institute of Systems Science, Chinese Academy of Sciences. He is an Associate Editor of Automatica and is an Associate Editor of the Asia Journal of Control, and Deputy Chief Editor or Associate Editor of four domestic journals. His research interests include nonlinear control systems, generalized Hamiltonian systems, and numerical method in systems and control.

Qiang Lu (F'03) graduated from the Graduate School of Tsinghua University, Beijing, China, in 1963

He is a faculty member of Tsingjua University. From 1984 to 1986, he was a Visiting Scholar and a Visiting Professor with Washington University, St. Louis, MO, and Colorado State University, Fort Collins, respectively, and from 1993 to 1995, he was a Visiting Professor with Kyushu Institute of Technology (KIT), Japan. He is now a Professor at Tsinghua University, and has been an Academician of the Chinese Academy of Science since 1991. His research interests are in nonlinear control theory and its applications to power systems. 